\title{
GDM alters paracrine regulation of feto-placental angiogenesis via the trophoblast
}

\author{
Jelena Loegl ${ }^{1}$, Erika Nussbaumer ${ }^{1}$, Silvija Cvitic ${ }^{1,2}$, Berthold Huppertz ${ }^{2}$, Gernot Desoye ${ }^{1}$ and Ursula Hiden ${ }^{1}$
}

Feto-placental angiogenesis and vascular development are tightly regulated by pro- and anti-angiogenic factors. Villous trophoblast may be a major source of these factors. It forms the classical placental barrier between mother and fetus, and is thus exposed to maternal influences as well. Metabolic and hormonal derangements in gestational diabetes mellitus (GDM) affect feto-placental angiogenesis and vascular growth. Here we hypothesized that GDM alters the trophoblast secretome, which will modulate the paracrine regulation of feto-placental angiogenesis. Primary term trophoblasts were isolated from normal $(n=6)$ and GDM $(n=6)$ pregnancies. Trophoblast conditioned medium (CM) was used to investigate paracrine effects of normal and GDM-exposed trophoblasts on feto-placental endothelial cells ( $\mathrm{fpECs} ; n=7$ ), using functional assays for 2D network formation, wound healing, chemotaxis, and proliferation. Gene expression of 23 pro- and anti-angiogenic factors was analyzed. Four trophoblast-derived paracrine regulators of angiogenesis were specifically measured in CM. CM from GDM trophoblasts increased 2D network formation of fpEC by 2.4-fold $(P<0.001)$, whereas wound healing was attenuated by 1.8 -fold $(P=0.02)$ and chemo-attraction to the CM was reduced by $33 \pm 9 \%(P=0.02)$. The effect of CM on proliferation was unchanged between normal and GDM trophoblasts. Expression analysis of pro- and anti-angiogenic molecules in normal and GDM trophoblasts revealed significant differences in ANGPT2, HGF, KISS1 and PLGF expression. Analysis of secreted proteins demonstrated reduced pigment epithelium derived factor and tumor necrosis factor- $a$ secretion by GDM trophoblasts. GDM alters the balance of trophoblast derived, angiogenesis modulating paracrine factors. This may contribute to GDM-associated changes in placental angiogenesis and vascular structure. Laboratory Investigation (2017) 97, 409-418; doi:10.1038/labinvest.2016.149; published online 23 January 2017

Angiogenesis is a multistep process including vascular sprouting, tubule morphogenesis, as well as adaption and stabilization of the vessel. ${ }^{1}$ The involved special cell functions such as chemotaxis, migration, contacting of other cells, and proliferation are tightly orchestrated. ${ }^{2}$ Thus, each sub-step of angiogenesis is target of regulation by autocrine, paracrine, and environmental factors. ${ }^{3}$

The placenta is a fetal organ between mother and fetus that transports oxygen, nutrients, and waste products. Therefore, the placenta is highly vascularized and feto-placental angiogenesis is tightly regulated by a complex interplay of pro- and anti-angiogenic factors. ${ }^{4,5}$ Moreover, the placental vasculature is plastic. To meet the growing demands of the fetus, placental modes of angiogenesis change throughout gestation: vasculogenesis starting in week three of pregnancy is followed by sprouting angiogenesis until mid-gestation. From week 25 on, primarily non-sprouting angiogenesis is thought to occur, ${ }^{4}$ although this clear switch was previously scrutinized. ${ }^{6}$ In parallel to these changing modes of placental angiogenesis throughout pregnancy, the composition of proand anti-angiogenic signals in the placenta change.

Many of these pro- and anti-angiogenic factors are produced and secreted by the villous trophoblast, ${ }^{7-12}$ a specialized placental cell type that covers the entire placental villous structure. The dynamic expression of angiogenic factors is evidenced by high trophoblast expression of vascular endothelial growth factor (VEGF) in early pregnancy, whereas later in pregnancy VEGF expression of villous trophoblast declines. ${ }^{7}$ Other trophoblast-derived angiogenesis modulating signals include fibroblast growth factor 2 (FGF2), ${ }^{8}$ soluble FMS-related tyrosine kinase 1 (sFLT1), ${ }^{9}$ placental growth factor (PGF), ${ }^{7}$ tumor necrosis factor- $\alpha$ (TNF- $\left.\alpha\right),{ }^{10}$ leptin, ${ }^{11}$ and KISS1 metastasis suppressor (KISS1). ${ }^{12}$ Recently, pigment epithelium derived factor (PEDF) was identified as a novel trophoblast-derived molecule regulating placental angiogenesis. ${ }^{13}$ The composition and interaction of these

Department of Obstetrics and Gynecology, Medical University of Graz, Graz, Austria and ${ }^{2}$ Institute of Cell Biology, Histology and Embryology, Medical University of Graz, Graz, Austria

Correspondence: Dr Ursula Hiden, Department of Obstetrics and Gynecology, Medical University of Graz, Auenbruggerplatz 14, 8036 Graz, Austria.

E-mail: ursula.hiden@medunigraz.at

Received 2 May 2016; revised 16 November 2016; accepted 22 November 2016 
trophoblast-derived factors will determine feto-placental angiogenesis. The trophoblast is exposed to and influenced by maternal endocrine and metabolic factors, which may in turn alter the trophoblast secretome. Thus, the maternal environment may influence the feto-placental vasculature as well.

Gestational diabetes mellitus (GDM) is defined as glucose intolerance that clinically manifests in the second half of gestation. ${ }^{14}$ It is associated with morphological changes of the placental vascular tree, that is, increased capillary branching ${ }^{15}$ and surface area. ${ }^{16,17}$ Calderon et al ${ }^{18}$ reported no change in vessel number, but a reduction in vessel diameter in GDM placenta. However, in placentas of mild hyperglycaemic women, they identified a pronounced increase in the number of vessels. So far, fetal metabolic and hormonal changes in response to maternal hyperglycemia ${ }^{19}$ have been regarded as a major mechanism for this GDM-associated placental vascular alterations. However, the maternal hyperglycemic, proinflammatory environment may also affect the expression of trophoblast-derived pro- and anti-angiogenic cytokines and growth factors. ${ }^{20}$ These in turn may contribute to GDMrelated changes of feto-placental angiogenesis.

Here we hypothesized that trophoblast-derived paracrine regulation of feto-placental angiogenesis differs in GDM compared with normal pregnancies. To test this hypothesis we isolated primary term trophoblasts from normal and GDM pregnancies, and analyzed the in vitro effect of their secretome on biological sub-processes involved in angiogenesis of primary feto-placental endothelial cells (fpECs) from normal, non-GDM pregnancies. As we found differences in these processes, we further analyzed gene expression and protein secretion of pro- and antiangiogenic factors.

Table 1 Subjects' characteristics of placenta donors for isolation of primary cells

\begin{tabular}{lccc}
\hline Subjects' characteristics & Control $(n=6)$ & GDM $(n=6)$ & $\begin{array}{c}\text { Significance } \\
(P \text {-value })\end{array}$ \\
\hline Maternal age (years) & $33.0 \pm 7.7$ & $33.2 \pm 5.6$ & 0.96 \\
Gestational age (weeks) & $40.3 \pm 1.1$ & $39.6 \pm 1.0$ & 0.39 \\
Maternal pre-pregnancy & $22.2 \pm 1.8$ & $24.1 \pm 3.8$ & 0.34 \\
BMl (kg/m²) & & & \\
Maternal weight & $15.1 \pm 2.3$ & $16.8 \pm 6.6$ & 0.66 \\
gain (kg) & & & \\
Maternal HbA1c (\%) & $\leq 6$ & $5.8 \pm 0.6$ & \\
Fetal weight (g) & $3452 \pm 424$ & $3470 \pm 408$ & 0.94 \\
Placental weight (g) & $678 \pm 144$ & $674 \pm 193$ & 0.92 \\
& & &
\end{tabular}

Abbreviations: BMI, body mass index; GDM, gestational diabetes mellitus.

\section{MATERIALS AND METHODS Ethical Approval}

The study was approved by the institutional review board and ethical committee of the Medical University of Graz (25-008 ex12/13). Informed consent of the patients was obtained.

\section{Sample Collection}

Placentas were collected after normal $(n=13)$ and GDM $(n=6)$ pregnancies, and trophoblasts and feto-placental endothelial cells were isolated. Control placentas were derived from pregnancies of non-smoking women, with a negative $75 \mathrm{mg}$ oral glucose tolerance test (oGTT), free of medical, or obstetrical disorders. All neonates had a birth weight between the 10th and 90th percentile. GDM was diagnosed at 28-32 weeks of gestation with a $75 \mathrm{~g}$ oGTT, with two or more values of the plasma glucose exceeding the following values: $92 \mathrm{~g} / \mathrm{l}$ fasting, $180 \mathrm{~g} / \mathrm{l}$ after $1 \mathrm{~h}$, and $153 \mathrm{~g} / \mathrm{l}$ after $2 \mathrm{~h}^{21}$ GDM women monitored their capillary glucose levels (memory reflectance meters; Accu-check, ROCHE Diagnostics) at least 4 times a day. They began appropriate diet providing $25-40 \mathrm{kcal} /$ day $/ \mathrm{kg}$ according to pre-pregnancy BMI. Compliance was checked by measuring maternal glycemia and fetal growth every 2 weeks. If mean maternal blood glucose values exceeded values of $1 \mathrm{~g} / \mathrm{l}$, insulin therapy was instituted $(n=2)$. All GDM patients were in good metabolic control with glycosylated $\mathrm{Hb}<5 \%$ and achieved target preprandial $<90 \mathrm{~g} / \mathrm{l}$ and postprandial $<119 \mathrm{~g} / \mathrm{l}$ glucose values until the last glucose control at the day before delivery. None of the women showed signs of hypertension or any other disease. Subjects' characteristics are shown in Table 1. The GDM women were similar in pre-pregnancy BMI with the control group and were metabolically well-controlled based on the HbAlc values. Both placental and birth weight were comparable with the non-GDM group.

\section{Isolation, Characterization and Culture of Human Placental Trophoblasts}

Trophoblasts were isolated as described by Chen et al. ${ }^{22}$ In brief, after mincing placental villous tissue from healthy controls $(n=6)$ and women with GDM $(n=6)$, the tissue was digested with a trypsin/dispase/DNase solution (Gibo, Roche, Sigma) for $90 \mathrm{~min}$. The cell suspension was centrifuged on a Percoll gradient (Sigma, St Louis, USA) at $4{ }^{\circ} \mathrm{C}$ for $30 \mathrm{~min}$ at $300 \mathrm{~g}$ without brakes. Trophoblast-enriched layers were purified by immunodepletion of contaminating cells using beads conjugated to MCA-81 antibody against HLA-A, B and C (Serotec, Puchheim, Germany). Trophoblasts $\left(3 \times 10^{6}\right)$ were seeded in each well of a six-well dish in $2 \mathrm{ml}$ DMEM containing $10 \% \mathrm{FCS}$ and $1 \%$ penicillin/streptomycin (Gibco, Lofer, Austria) at $37^{\circ} \mathrm{C}$ and $8 \%$ oxygen. After $48 \mathrm{~h}$, viability was tested by measuring secreted $\beta$-hCG levels in the culture supernatant (Siemens). Purity was determined by immunocytochemical staining for the trophoblast marker cytokeratin 7 (Dako, Vienna, Austria) and for the mesenchymal cell 
marker vimentin (Dako). ${ }^{23}$ Only preparations with a purity $\geq$ $99 \%$ were used.

\section{Preparation of CM}

Trophoblasts were isolated from six different control placentas and six GDM placentas (Table 1). Twenty hours after isolation, medium was changed to $2 \mathrm{ml}$ of DMEM (Gibco) and endothelial basal medium (EBM, Clonetics, Lonza) mixed 1:1 with 7.5\% FCS, further referred to as DE medium. The CM was collected after another $48 \mathrm{~h}$, centrifuged at $300 \mathrm{~g}$ for $5 \mathrm{~min}$ and frozen at $-80{ }^{\circ} \mathrm{C}$ in aliquots. For functional assays, two pools of CM from normal and GDM trophoblast isolations were produced. Each of the two control and two GDM pools consisted of three individual CM. Pooling was necessary to obtain sufficient volume for testing in replicates.

\section{Isolation, Characterization and Culture of Human Feto-Placental Endothelial Cells}

Primary feto-placental endothelial cells (fpECs) were isolated from third trimester placenta $(n=7)$ after normal, uncomplicated full-term pregnancy following the protocol established in our laboratory. ${ }^{24}$ Dissected arterial chorionic vessels were washed with Hanks balanced salt solution (HBSS; Gibco). Endothelial cells were isolated by perfusion of vessels with a prewarmed $\left(37^{\circ} \mathrm{C}\right)$ enzymatic solution $(0.1 \mathrm{U} / \mathrm{ml}$ collagenase and $0.8 \mathrm{U} / \mathrm{ml}$ dispase; Roche) in HBSS for $8 \mathrm{~min}$. After centrifugation $(200 \mathrm{~g})$ for $5 \mathrm{~min}$, cells were resuspended in EBM supplemented with EGM-MV Bullet Kit (Clonetics, Lonza). Isolated fpEC were seeded in culture flasks coated with $1 \%$ gelatin (Sigma-Aldrich) and expanded at $37^{\circ} \mathrm{C}$ and $12 \%$ oxygen. The purity of the fpEC isolations was characterized by immunocytochemical staining for the endothelial cell marker von Willebrand factor and for fibroblast-specific antigen and smooth muscle actin, both potentially contaminating cell types. ${ }^{24}$ Isolated cells were cultured and used for experiments up to passage 5. Two days before functional assays, cells were cultured at $8 \%$ oxygen.

\section{D Network Formation Assay}

To assess the ability of human fpEC to form tube-like structures, trypsinized fpEC $\left(1 \times 10^{4}\right)$ were resuspended in $100 \mu \mathrm{l} \mathrm{CM}$ from normal and GDM trophoblasts. This cell suspension was seeded on growth factor reduced Matrigel (BD Bioscience, Vienna, Austria). Network formation started immediately after attachment of the cells on the Matrigel and was monitored in a cell observer (Carl Zeiss Imaging Solutions $\mathrm{GmbH}$, Munich, Germany). Images of each well were captured every hour over a period of $24 \mathrm{~h}$. Total tube length was quantified with ImageJ software (NIH) using the AngioJ-Matrigel assay plugin, kindly provided by Diego Guidolin, University of Padova, Italy. As all measured parameters showed a similar trend (not shown), total tube length was used as representative measure. All experiments were carried out in triplicates using seven different fpEC isolations.

\section{Wound-Healing Assay/Migration assay}

A wound-healing assay evaluated directional migration of fpEC treated with CM of normal or GDM trophoblasts. Cell culture inserts for wound-healing assay (Ibidi GmbH, Planegg, Germany) were placed in wells of a 12 -well plate. Cells $\left(3.5 \times 10^{4} / 70 \mu \mathrm{l} \mathrm{EBM}\right)$ were seeded in each of the two compartments of the inserts. After $24 \mathrm{~h}$, inserts were removed leaving a cell monolayer separated by a $500 \mu \mathrm{m}$ gap and the medium was replaced by CM of normal or GDM trophoblasts. Cell migration was monitored at $37^{\circ} \mathrm{C}$ and $8 \%$ oxygen for $24 \mathrm{~h}$, with a picture taken every $30 \mathrm{~min}$ (cell observer; Zeiss). The gap closure was quantified with AxioVision LE64 software (Zeiss) after 3, 6, 12 and $18 \mathrm{~h}$. All experiments were carried out in triplicates using six different fpEC isolations. Seven pictures per well were used for quantification.

\section{Chemoattraction Assay}

The chemoattraction of fpEC towards CM of normal or GDM trophoblasts was tested with a 96-well chemotaxis chamber system (Neuroprobe, Warwick, UK). FpEC were serum starved for $3 \mathrm{~h}$ in EBM without supplements. Then, $1 \times 10^{4}$ cells were resuspended in control medium and seeded on fibronectin-coated polycarbonate filters $(8 \mu \mathrm{m}$ pores). CM was placed in the lower chambers of the microplates and the filter was put on top. FpEC could migrate through the pores towards the $\mathrm{CM}$ for $4 \mathrm{~h}$ at $37^{\circ} \mathrm{C}$ and $8 \%$ oxygen. The upper surface of the filter was wiped clean of non-migrating cells. Migrated cells were fixed with $4 \%$ formaldehyde for $15 \mathrm{~min}$ and stained with DAPI $(2.5 \mu \mathrm{g} / \mathrm{ml}$; Invitrogen, Vienna, Austria) for $1 \mathrm{~min}$. Pictures were taken with a digital camera attached to an inverted phase-contrast microscope (Zeiss). From each filter well 35 pictures were taken. Out of these, seven pictures were randomly selected and analysed using DotCount v1.2 (online provided by Martin Reuter, MIT). All experiments were carried out in triplicates using six different fpEC isolations.

\section{Proliferation Assay}

The effect of $\mathrm{CM}$ on fpEC proliferation was analyzed by measuring the incorporation of bromodeoxyuridine (BrdU) into DNA with Cyclex BrdU ELISA Kit (Cyclex, Woburn, USA), according to manufacturer's instructions. Cells $\left(6 \times 10^{3} / 100 \mu \mathrm{l} \mathrm{EBM}\right)$ were seeded in wells of a 96-well plate. After $20 \mathrm{~h}$, cells reached $70 \%$ confluence. Then, medium was replaced by CM from normal or GDM trophoblasts and fpEC were cultured for further $24 \mathrm{~h}$ at $37^{\circ} \mathrm{C}$ and $8 \%$ oxygen. Then, BrdU was added to a final concentration of $10 \mu \mathrm{M}$ and cells were incubated for $2 \mathrm{~h}$. After fixation, an anti-BrdU antibody was added, followed by incubation with a HRP-conjugated secondary antibody. Absorbance was measured at 450/540 nm using the FluoSTAR Optima 413 plate-reader (BMG Lab 
Table 2 Differences in expression of genes encoding pro- and anti-angiogenic factors between normal and GDM-derived TB

\begin{tabular}{|c|c|c|c|c|c|}
\hline Gene symbol & Gene name & Taqman assay ID & FC vs normal TB & Sign. & Major angiogenic effect \\
\hline ANGPT1 & Angiopoietin 1 & Hs00375822_m1 & ND & & pro \\
\hline CSF2 & Colony-stimulating factor 2 & Hs00929873_m1 & ND & & pro \\
\hline CSF3 & Colony-stimulating factor 3 & Hs00738432_g1 & $2.4 \pm 0.6$ & 0.520 & pro \\
\hline HGF & Hepatocyte growth factor & Hs00300159_m1 & $2.2 \pm 0.5$ & 0.010 & pro \\
\hline$|G F|$ & Insulin-like growth factor 1 & Hs01547656_m1 & $1.7 \pm 0.3$ & 0.107 & pro \\
\hline IGF2 & Insulin-like growth factor 2 & Hs04188276_m1 & $0.8 \pm 0.2$ & 0.511 & pro \\
\hline 128 & Interleukin 8 & Hs00174103_m1 & $1.0 \pm 0.5$ & 0.974 & pro \\
\hline KISS1 & KISS1 metastasis inhibitor & Hs00158486_m1 & $5.8 \pm 1.2$ & 0.010 & anti \\
\hline PEDF & Pigment epithelium-derived factor & Hs01106937_m1 & $1.4 \pm 0.2$ & 0.110 & anti \\
\hline PGF & Placental growth factor & Hs00182176_m1 & $6.2 \pm 1.8$ & 0.006 & pro \\
\hline$P R L$ & Prolactin & Hs00168730_m1 & ND & & pro \\
\hline TGFA & Transforming growth factor $a$ & Hs00608187_m1 & $0.9 \pm 0.2$ & 0.268 & pro \\
\hline TGFB1 & Transforming growth factor $\beta 1$ & Hs00998133_m1 & $4.2 \pm 3.1$ & 0.300 & pro/anti \\
\hline THBS1 & Thrombospondin 1 & Hs00962908_m1 & $1.2 \pm 0.4$ & 0.538 & anti \\
\hline THBS2 & Thrombospondin 2 & Hs01568063_m1 & ND & & anti \\
\hline TIMP3 & Tissue inhibitor of metalloproteinase 3 & Hs00165949_m1 & $1.3 \pm 0.2$ & 0.186 & anti \\
\hline TNFA & Tumor necrosis factor $a$ & Hs01113624_g1 & $-3.8 \pm 0.1$ & 0.033 & pro \\
\hline
\end{tabular}

Abbreviations: FC, fold change; GDM, gestational diabetes mellitus; ND, not detected after 35 amplification cycles; sign., significance; TB, trophoblasts. Data are given as FC in gene expression between GDM-derived TB vs normal TB \pm s.e.m. Significantly different expressed genes are printed in bold.

Technologies, Offenburg, Germany). Experiments were carried out in triplicates using five different fpEC isolations.

\section{Lactate Dehydrogenase Assay}

Cytotoxicity of the CM on fpEC was investigated by lactate dehydrogenase (LDH) assay (Takara, Japan) according to the manufacturer's protocol. Cells $\left(6 \times 10^{3} / 100 \mu \mathrm{l} \mathrm{EBM}\right)$ were seeded in wells of a 96-well plate. After $20 \mathrm{~h}$, medium was replaced by CM from normal or GDM trophoblasts and fpEC were incubated for another $24 \mathrm{~h}$ at $37^{\circ} \mathrm{C}$ and $8 \%$ oxygen. Absorbance was measured immediately thereafter at 490 $1650 \mathrm{~nm}$ using a microplate reader (spectroMax 250 Molecular Devices MWG-Biotech, Germany). Experiments were carried out in triplicates using five different fpEC isolations.

\section{RNA isolation, cDNA Synthesis and Quantitative Reverse-Transcription PCR}

Total RNA was isolated immediately after collection of CM using the RNeasy mini Kit (Qiagen, Hilden, Germany) according to manufacturer's instructions. RNA concentration and purity was assessed with a BioPhotometer (Eppendorf, Vienna, Austria). Total RNA (500 ng) was used to synthesize cDNA with Superscript II reverse transcriptase (Invitrogen), following the manufacturer's protocol. Quantitative reversetranscription PCR reaction was performed with TaqMan gene expression assays (Applied Biosystems, Vienna, Austria; Table 2) in an AB7900 Syllabus (Applied Biosystems). Gene expression was normalized to the mean expression values of the two housekeeping genes HPRT1 (Hs02800695_m1) and RPL30 (Hs00265497_m1). The $2^{-\Delta \Delta \mathrm{ct}}$ method was used to calculate the fold change.

\section{Quantification of Proteins in CM}

Angiogenic factors in CM of normal and GDM trophoblasts were quantified according to the manufacturer's instructions using ELISA development kits (PeproTech, Vienna, Austria) for TNF $\alpha$ (900-M25) and FGF2 (900-M08) with the appropriate Buffer Kit (900-K00). FGF2 concentrations were determined after volume reduction to $50 \%$, ie, an enrichment by twofold. The concentrations of sFLT1 and PEDF were 
a

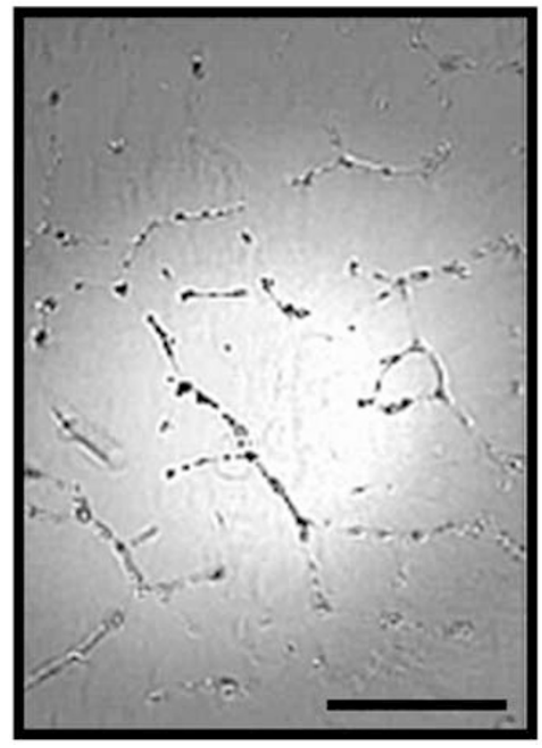

dTB CM

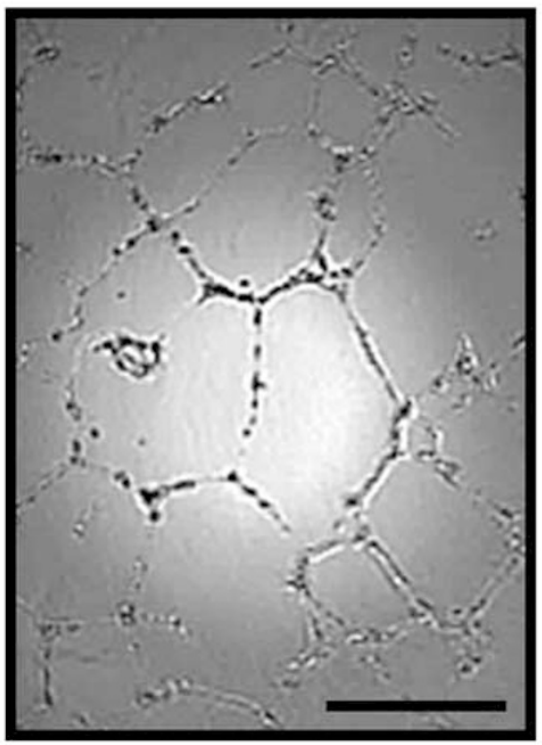

b

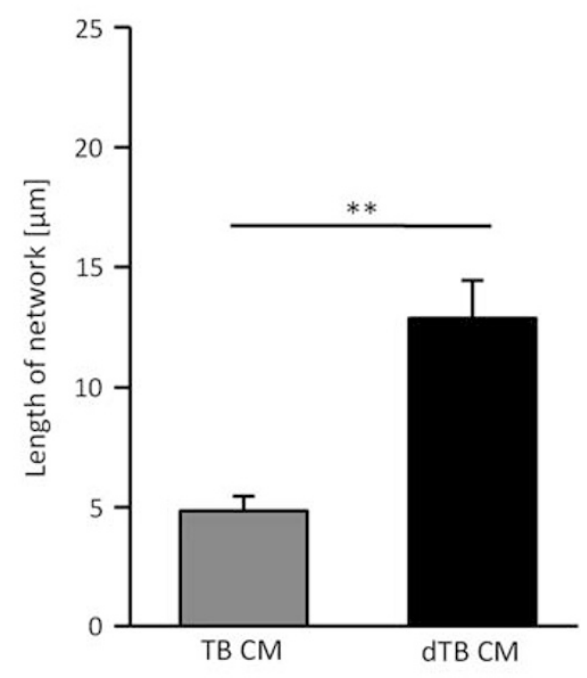

Figure 1 Paracrine effect of conditioned media from normal vs gestational diabetes mellitus (GDM)-derived trophoblasts on network formation of fpEC. (a) Representative images of $2 \mathrm{D}$ networks of fpEC exposed to conditioned medium (CM) of normal trophoblasts (TB) and GDM-derived trophoblasts (dTB) after $12 \mathrm{~h}$ on growth factor-reduced Matrigel. (b) The total tube length $(\mu \mathrm{m})$ was analyzed after $12 \mathrm{~h}$ with ImageJ software. Data are given as mean \pm s.e.m. of $n=7 \mathrm{fpEC}$ isolations, each performed in triplicates. CM of $n=6$ TB and $n=6$ dTB isolations were pooled, with the two control pools and the two dTB pools containing three individual CM each. ${ }^{* *} P<0.01$. Scale bars, $250 \mu \mathrm{m}$.

measured using immunoassays (Biovendor-R\&D Products, Minneapolis, USA) according to the manufacturer's instructions. As a cell-free medium control, DE with $7.5 \%$ FCS was incubated, collected, and stored under the same conditions.

\section{Statistical Analysis}

After testing for normal distribution (Shapiro-Wilk), Student's $t$-test was used. Analysis of functional effects in fpEC used paired $t$-test. Significances were accepted if $P<0.05$.

\section{RESULTS}

\section{Paracrine Effects on Feto-Placental Endothelial Cells} Differ Between Normal and GDM Exposed Trophoblasts FpECs were seeded on growth factor reduced Matrigel and exposed to medium conditioned by primary trophoblasts isolated after normal or GDM pregnancy. Networks developed immediately after seeding of the cells and were observed over a period of $24 \mathrm{~h}$. After $12 \mathrm{~h}$, networks were fully developed and were more pronounced, ie, 2.4 -fold longer tubes $(12.85 \pm 1.58$ vs $4.81 \pm 0.64 \mu \mathrm{m} ; P<0.001)$, after $12 \mathrm{~h}$ with $\mathrm{CM}$ of GDM than $\mathrm{CM}$ of normal trophoblasts (Figures $1 \mathrm{a}$ and $\mathrm{b}$ ).

The effect of CM from normal and GDM trophoblasts on fpEC migration was tested using a wound-healing and a chemotaxis assay. In the wound-healing assay, cell migration into a defined gap $(500 \mu \mathrm{m})$ was observed over a period of $12 \mathrm{~h}$. After $12 \mathrm{~h}$ gap width was 1.8 -fold $(P=0.02)$ larger in size with $\mathrm{CM}$ from GDM than normal trophoblasts, suggesting slower migration (Figures $2 \mathrm{a}$ and $\mathrm{b}$ ). The chemoattraction assay measured fpEC migration through an extracellular matrix towards the different CM. CM of GDM trophoblasts revealed $33 \pm 8 \%(P=0.02)$ reduced chemoattraction $v s \mathrm{CM}$ of control trophoblasts (Figure 2c).

Proliferation as a further process needed for angiogenesis in vivo was measured by BrdU incorporation. It did not differ between fpEC treated with CM of normal vs GDM trophoblasts (Figure 3a). Release of $\mathrm{LDH}$ by fpEC as a measure of CM cytotoxicity did not differ between CM of normal and GDM exposed trophoblasts (Figure 3b).

\section{Gene Expression and Protein Secretion of Pro- and Anti-Angiogenic Factors Differ in GDM-Exposed Trophoblasts}

The different effect of CM from normal vs GDM trophoblasts on fpEC functions suggests that GDM alters the trophoblast secretome. To test this, 23 pro- and anti-angiogenic factors were selected based on the literature ${ }^{25-36}$ and analyzed on gene expression level. Three of these factors were additionally measured on protein level and sFLT1 secretion was quantified only in the CM.

Transcript levels of five mRNAs differed between normal and GDM trophoblasts, ie, the anti-angiogenic KISS1 (5.2-fold; $P<0.05$ ) and was increased, as well as ANGPT2 (2.5-fold; $P<0.05)$ and the pro-angiogenic HGF $(2.0$-fold; $P<0.05)$ and $P G F$ (5.6-fold; $P<0.01)$. TNFA was reduced $(-3.8$-fold; $P<0.05)$ (Table 2). Expression of ANGPT1, CSF2, $P R L$ and THBS2 was below detection limit after 35 amplification cycles, whereas expression of the other factors remained unchanged. Notably, also expression of VEGFA was 
unchanged. Analysis of protein secretion of TNF $\alpha$ and FGF2 as important placental pro-angiogenic factors confirmed the mRNA results: TNF $\alpha$ was reduced (sixfold; $P=0.008$ ) and FGF2 unchanged (Figures $4 \mathrm{a}$ and b). Moreover, we measured secretion of PEDF that we recently discovered as a trophoblast-derived anti-angiogenic factor ${ }^{13}$ and sFLT1, a soluble splice variant of the VEGF receptor FLT1, which captures VEGF was not measured on mRNA levels. In

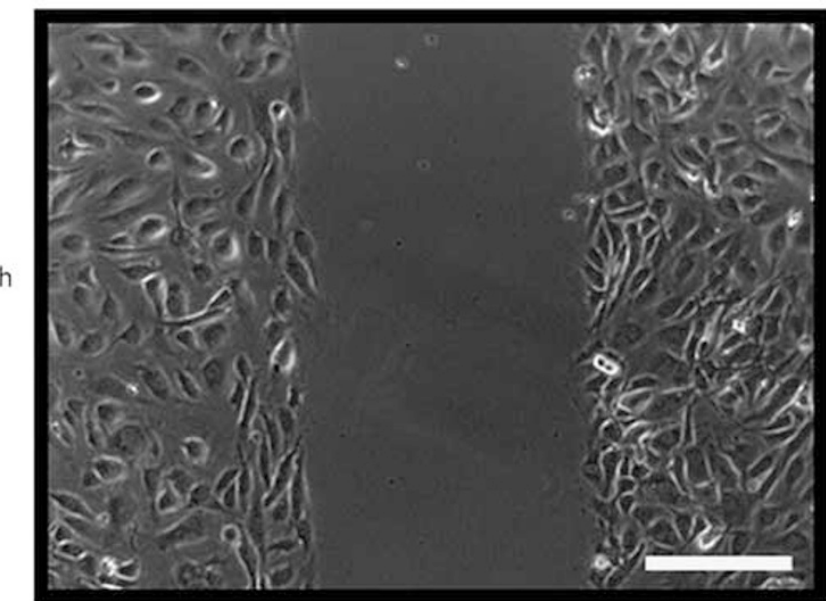

$12 \mathrm{~h}$

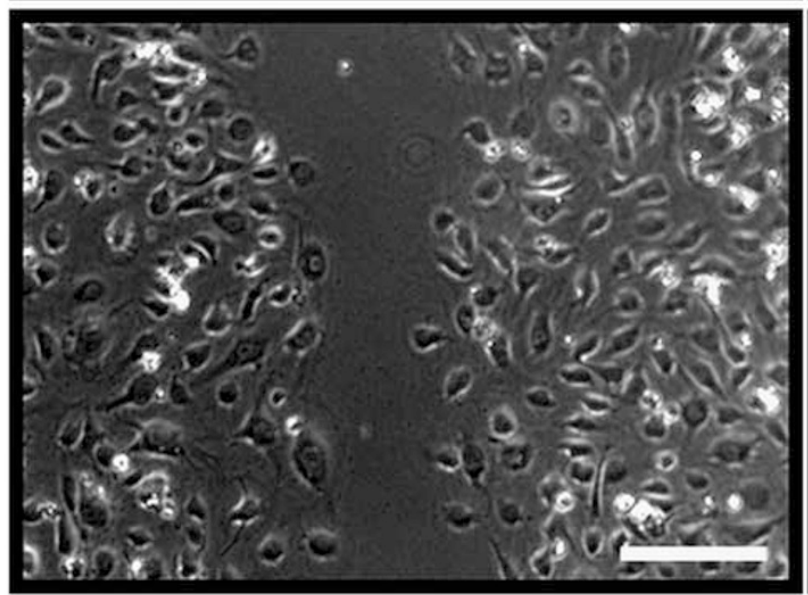

b

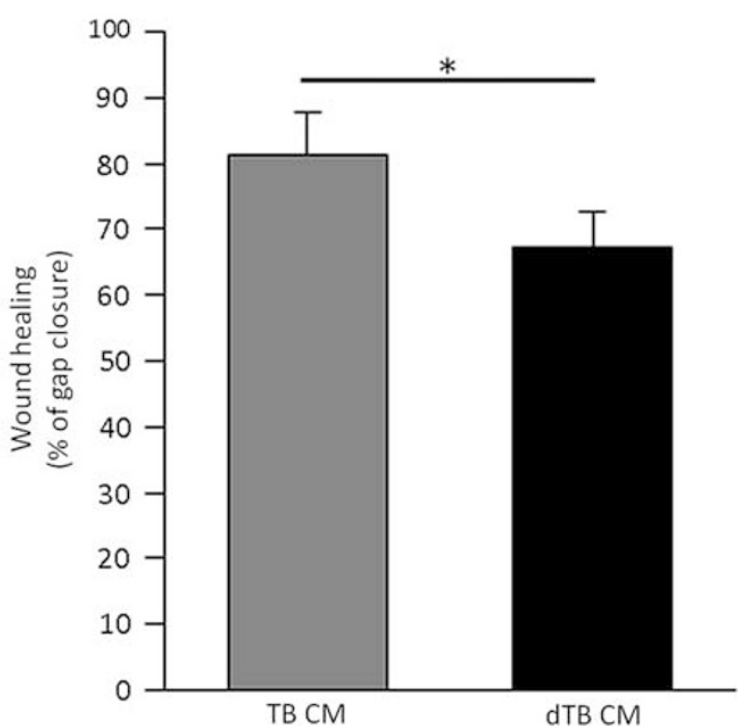

dTB CM
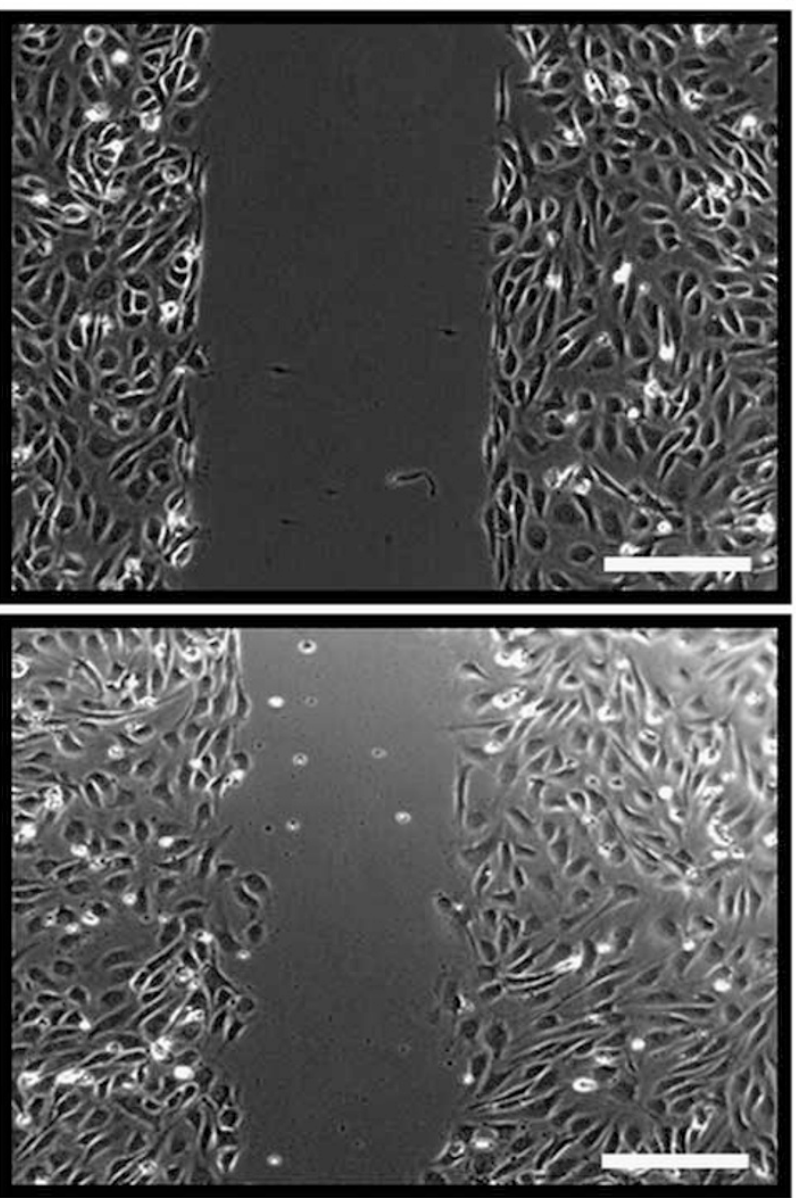

C

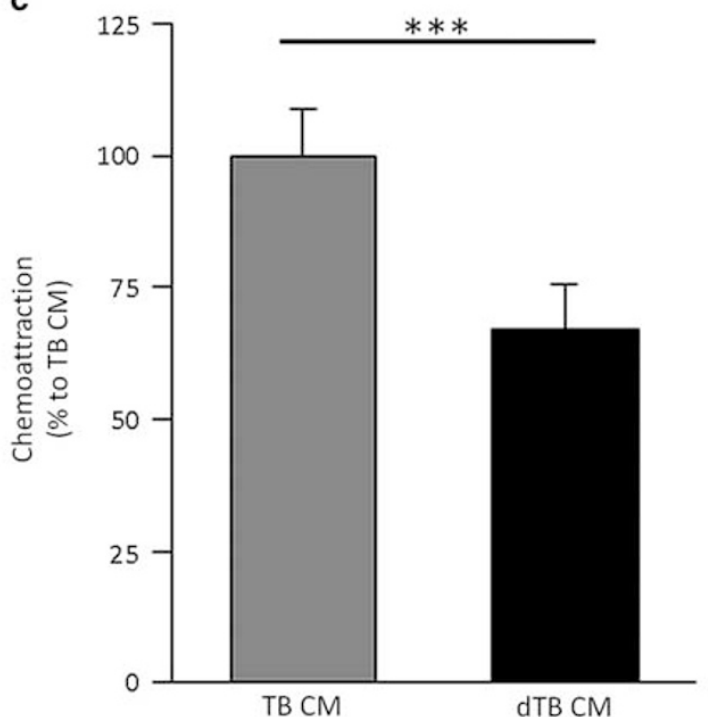


contrast to unchanged mRNA levels, PEDF secretion was decreased in GDM trophoblasts by $65 \pm 11 \%(P=0.024)$. Secretion of sFLT1 was unchanged (Figures $4 c$ and $d$ ).

\section{DISCUSSION}

This study investigated whether GDM alters the paracrine regulation of feto-placental endothelial function by the trophoblast. Main findings were that GDM modifies the trophoblast secretome and, consequently, influences the paracrine effect of trophoblasts on in vitro behavior of primary fpEC.

Angiogenesis is a complex and tightly regulated process. It involves the escape of quiescence, chemotaxis, migration, tubulogenesis, and vessel stabilization. ${ }^{1}$ These processes depend on regulation by cell-cell interaction, extracellular matrix composition, and soluble factors. ${ }^{3}$ We here focussed on trophoblast-derived soluble factors and whether their effect on fpEC is altered in maternal GDM. Therefore, we used functional in vitro assays to determine the effect of these trophoblast-derived factors on distinct aspects of angiogenesis: 2D network formation, wound healing, chemotaxis, and proliferation. The functional assays revealed that different aspects of angiogenesis responded differently to the changed paracrine action of trophoblast in GDM: although CM of GDM-exposed trophoblasts induced more 2D network formation, wound healing and chemo-attraction toward the CM was reduced by paracrine factors of GDM-exposed trophoblasts. This may seem controversial, as all these processes depend on cell movement. However, these processes reflect distinct events of angiogenesis: The 2D network formation assay employed in this study measures cell reorganisation, contacting of scattered endothelial cells with a

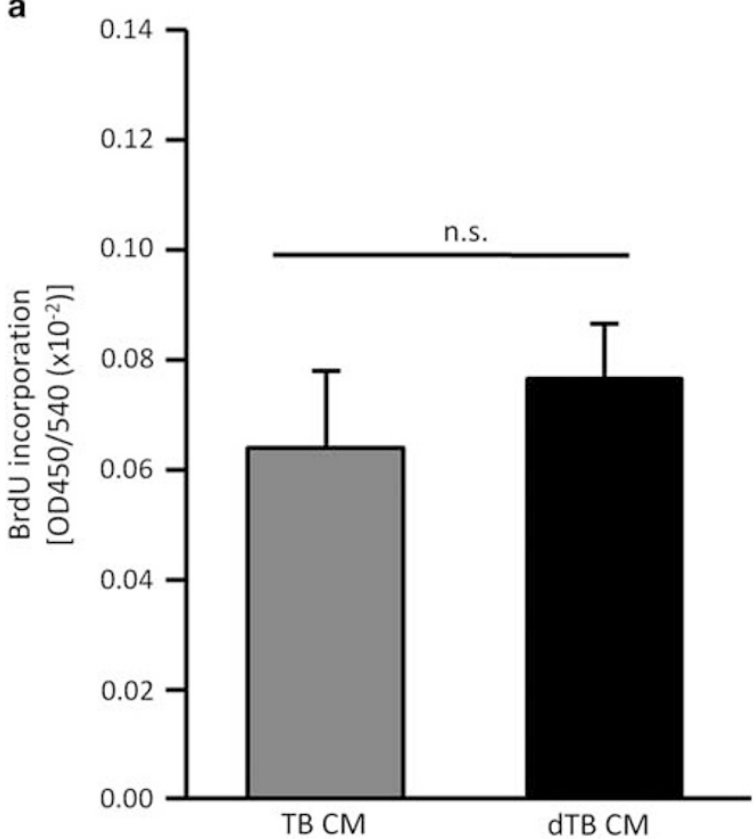

b

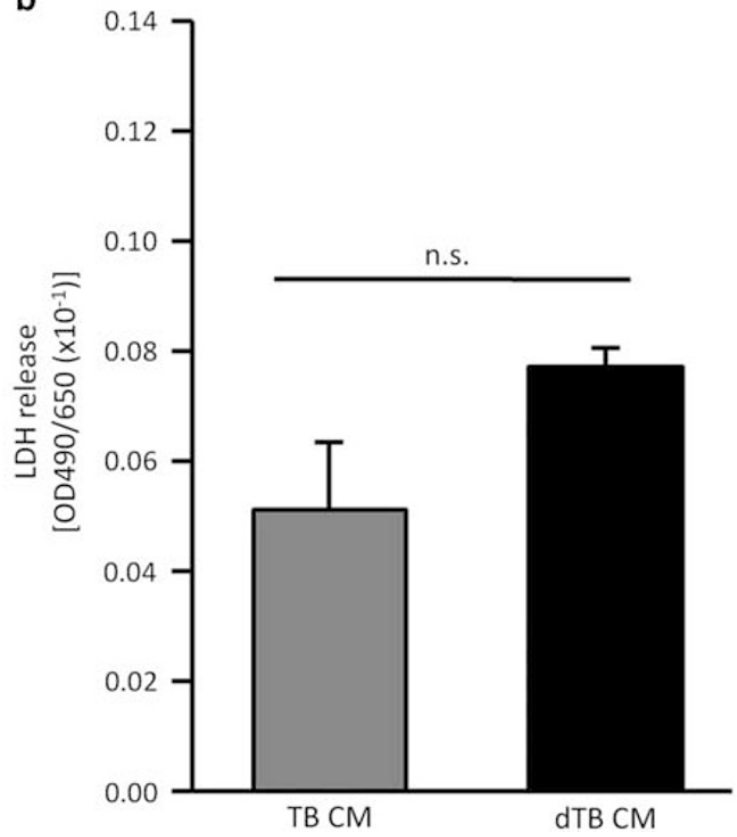

Figure 3 Paracrine effect of conditioned media from normal vs gestational diabetes mellitus (GDM)-derived trophoblasts on proliferation and cytotoxicity of fpEC. (a) Bromodeoxyuridine (BrdU) incorporation into DNA of fpEC treated with conditioned medium (CM) of normal trophoblasts (TB) and GDM-derived trophoblasts (dTB) was determined by ELISA. Data are given as mean \pm s.e.m. of $n=5 \mathrm{fpEC}$ isolations, each measured in triplicates. (b) Lactate dehydrogenase (LDH) release of fpEC after $24 \mathrm{~h}$ culture was unchanged between CM from normal and GDM derived trophoblasts. Data are given a mean \pm s.e.m. of $n=5 \mathrm{fpEC}$ isolations, each measured in triplicates. CM of $n=6 \mathrm{~TB}$ and $n=6 \mathrm{dTB}$ isolations were pooled, with the two control pools and the two dTB pools containing three individual CM each. n.s., not significant.

Figure 2 Paracrine effect of conditioned media from normal vs gestational diabetes mellitus (GDM)-derived trophoblasts on wound healing and chemotaxis of fpEC. (a) Representative images of fpEC treated with conditioned medium (CM) of normal trophoblasts (TB) and GDM-derived trophoblasts (dTB) in a wound-healing assay after 1 and $12 \mathrm{~h}$. (b) Quantitative analysis of wound-healing assay after $12 \mathrm{~h}$. Data give reduction in gap size relative to the original size of the gap as mean \pm s.e.m. of $n=5 \mathrm{fpEC}$ isolations, each performed in triplicates. (c) Migration of fpECs through a fibronectin coated filter toward CM of normal and GDM-derived trophoblasts was analyzed after $4 \mathrm{~h}$ with DotCount Software by counting the number of DAPI stained cells. Data are given as mean \pm s.e.m., each measured in triplicates of $n=6 \mathrm{fpEC}$ isolations. CM of $n=6$ TB and $n=6 \mathrm{dTB}$ isolations were pooled, with the two control pools and the two dTB pools containing three individual $\mathrm{CM}$ each. ${ }^{*} P<0.05$ and ${ }^{* * *} P<0.001$. Scale bars $=500 \mu \mathrm{m}$. 
a

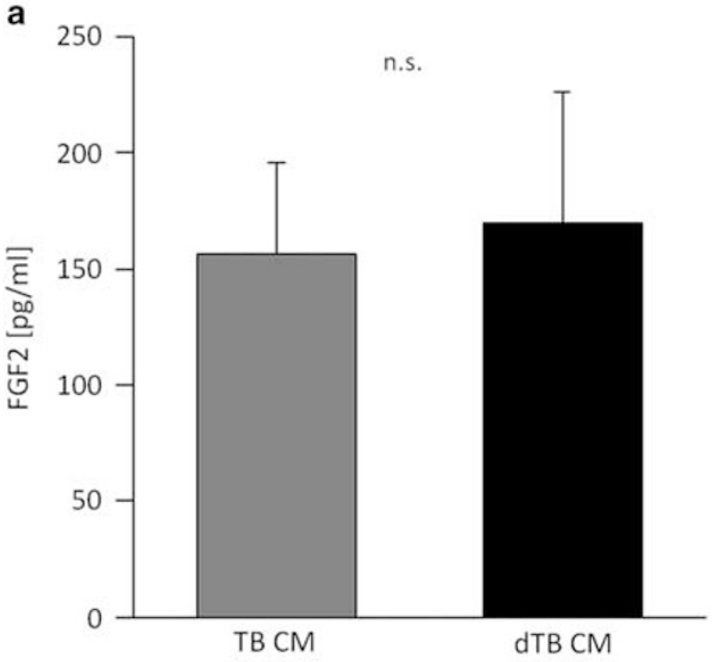

C

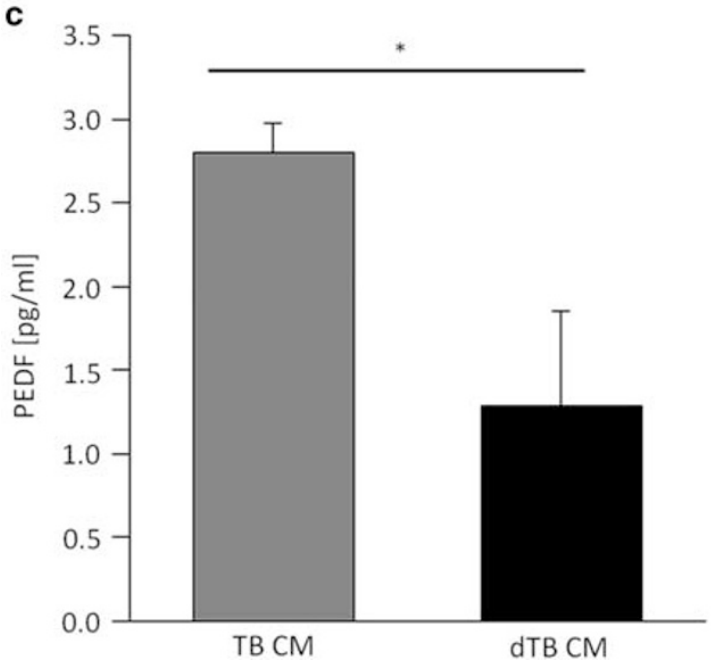

b

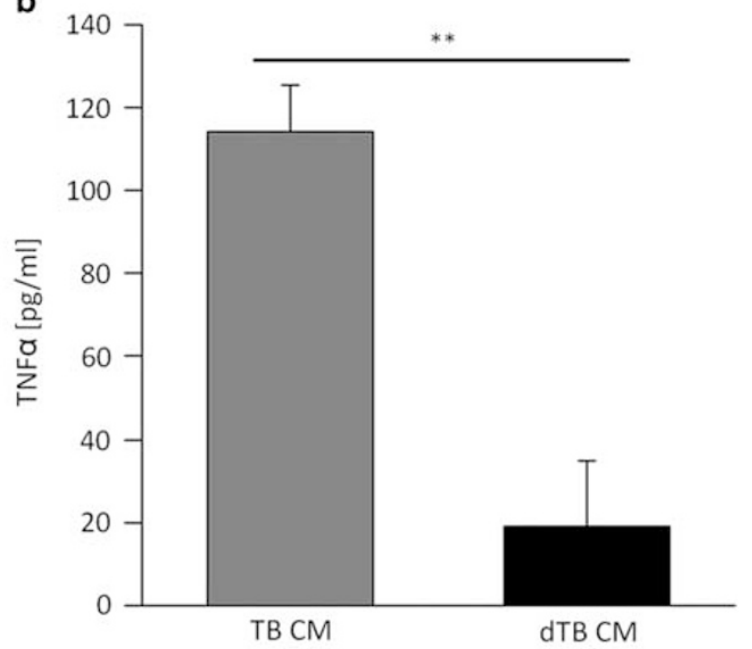

d

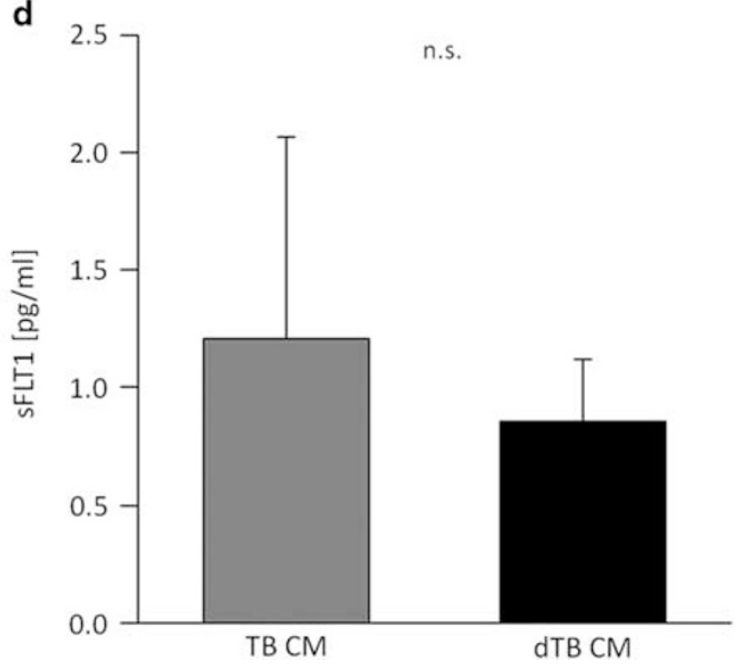

Figure 4 Secretion of pro- and anti-angiogenic factors by trophoblasts isolated after normal and gestational diabetes mellitus (GDM) pregnancy. Secretion of the pro-angiogenic fibroblast growth factor 2 (FGF2) (a) and tumor necrosis factor- $a$ (TNFa) (b), and of the anti-angiogenic pigment epithelium derived factor (PEDF) (c) and soluble FMS-related tyrosine kinase 1 (sFLT1) (d) by trophoblasts isolated after normal (TB) and GDM pregnancy (dTB), determined by ELISA. Data are given as mean \pm s.e.m. of $n=6$ individual conditioned medium (CM) from both control and GDM-derived trophoblast isolations, each measured in duplicates. ${ }^{*} P<0.05$ and ${ }^{*} P<0.01$, n.s., not significant. In non-conditioned medium, levels of the factors were below the detection limit of the ELISAs.

each other and the formation of cord-like structures in between of them, whereas the wound-healing assay determines migration of cells from a confluent cell monolayer into a cell-free area. Chemo-attraction assays measures chemotaxis towards an attractant, ie, the trophoblast CM.

Moreover, the presence of VEGF in the Matrigel may underlie the different effects of CM from GDM-exposed trophoblasts on network formation $v s$ migration. On Matrigel, CM of GDM-derived trophoblast stimulated network formation, in absence of Matrigel, CM of GDM derived trophoblast reduced migration. Matrigel, even when growth factor reduced, is an extracellular matrix rich in growth factors such as VEGFA. Expression of ANGPT2 was increased in GDM-exposed trophoblasts and secretion of PEDF decreased. Both factors induce distinct effects depending on the presence or absence of VEGFA: effects of ANGPT2 promotes angiogenesis only in the presence of VEGFA 37,38 and PEDF inhibits angiogenesis only when cells are stimulated by surrounding VEGFA ${ }^{13}$. Thus, VEGFA present in the extracellular matrix may alter the angiogenic response to the different CM.

Surprisingly, we did not find elevated levels of VEGFA expressed by GDM trophoblast and also secretion of the VEGF capture molecule sFLT1 was unchanged. Nevertheless, a role of VEGFA signaling in altered paracrine effects of GDM trophoblasts on fpEC may be hypothesized, as some of the factors altered in GDM trophoblast interact and affect VEGF signaling: the downregulated PEDF attenuates VEGFA 
signaling through the VEGFA receptor kinase insert domain receptor (KDR). ${ }^{13,39}$ PGF expression is upregulated by GDM. PGF protein induces angiogenesis by binding to the VEGF receptor FLT1 and, by activation of FLT1, even stimulates VEGFA-induced KDR activation. ${ }^{40}$ Thus, the reduction of PEDF and the increase of PGF may promote VEGFA-KDRinduced angiogenesis, albeit unchanged VEGFA levels, and thus stimulate angiogenic events in the GDM placenta.

These changes in expression and secretion of angiogenesis regulating factors may parallel the in vivo findings of placental vascular changes in GDM: Some studies demonstrate increased number of placental vessels in GDM, ${ }^{16,17}$ whereas another study found higher vascularisation only in cases of mild hyperglycemia with normal oGTT but not in the GDM group. ${ }^{18}$ However, one needs to keep in mind that the paracrine regulation of feto-placental angiogenesis by trophoblast signals is only one level of control with fetal and other placental signals representing a second level, ${ }^{13}$ which will also affect feto-placental vascular architecture. Such fetal and placental signals include circulating fetal pro- and antiangiogenic factors, as well as paracrine factors secreted by, for instance, Hofbauer cells. Thus, we cannot directly translate the altered in vitro effects of medium conditioned by GDM trophoblasts to placental vascular changes occurring in GDM in vitro, as trophoblast-derived signals will be integrated with other pro- and anti-angiogenic signals.

Overall, our data demonstrate that GDM disturbs trophoblast-derived paracrine pro- and anti-angiogenic signals and that, via secreted paracrine signals, the trophoblast contributes to the changes in the feto-placental vascular network in GDM. The trophoblast is exposed to the maternal environment, ie, circulating physiologically active substances and metabolites, as well as cytokines and growth factors secreted by decidual cells including NK cells, dendritic cells, and decidual macrophages. This opens the possibility that the mother, through effects on the trophoblast, can indirectly influence biological processes ${ }^{41}$ such as angiogenesis at the feto-placental interface.

In vivo the villous trophoblast layer represents a polarized syncytium with a subjacent pool of proliferating and differentiating cells. This specialized structure does not develop after isolation in vitro, thus precluding analysis of the direction of secretion, ie, into the maternal circulation or through the basement membrane into either the placental stroma or directly to the endothelial cells. However, as CM of short-term trophoblast cultures were used, it is tempting to speculate that this $\mathrm{CM}$ is derived from mononucleated cytotrophoblasts that secrete toward the placental stroma and do not have any direct contact to maternal blood.

In conclusion, our data show that the balance of angiogenesis regulating factors secreted by trophoblasts differs in GDM. This imbalance will affect the feto-placental endothelium and thus may contribute to the altered fetoplacental vascular architecture in GDM.
ACKNOWLEDGMENTS

This study was financially supported by the city of Graz (A16-013351/2009).

\section{DISCLOSURE/CONFLICT OF INTEREST}

The authors declare no conflict of interest.

1. Irvin MW, Zijlstra A, Wikswo JP, et al. Techniques and assays for the study of angiogenesis. Exp Biol Med (Maywood) 2014;239:1476-1488.

2. Betz $C$, Lenard A, Belting HG, et al. Cell behaviors and dynamics during angiogenesis. Development 2016;143:2249-2260.

3. Conway EM, Collen D, Carmeliet P. Molecular mechanisms of blood vessel growth. Cardiovasc Res 2001;49:507-521.

4. Kaufmann P, Mayhew TM, Charnock-Jones DS. Aspects of human fetoplacental vasculogenesis and angiogenesis. II. Changes during normal pregnancy. Placenta 2004;25:114-126.

5. Charnock-Jones DS, Kaufmann P, Mayhew TM. Aspects of human fetoplacental vasculogenesis and angiogenesis. I. Molecular regulation. Placenta 2004;25:103-113.

6. Burton GJ, Charnock-Jones DS, Jauniaux E. Regulation of vascular growth and function in the human placenta. Reproduction 2009;138: 895-902.

7. Vuorela P, Hatva E, Lymboussaki A, et al. Expression of vascular endothelial growth factor and placenta growth factor in human placenta. Biol Reprod 1997;56:489-494.

8. Arany $E$, Hill DJ. Fibroblast growth factor-2 and fibroblast growth factor receptor-1 mRNA expression and peptide localization in placentae from normal and diabetic pregnancies. Placenta 1998;19: 133-142.

9. Furuya $\mathrm{M}$, Okuda $\mathrm{M}$, Usui $\mathrm{H}$, et al. Expression of angiotensin II receptorlike 1 in the placentas of pregnancy-induced hypertension. Int J Gynecol Pathol 2012;31:227-235.

10. Chen $\mathrm{HL}$, Yang YP, Hu XL, et al. Tumor necrosis factor alpha mRNA and protein are present in human placental and uterine cells at early and late stages of gestation. Am J Pathol 1991;139:327-335.

11. Henson MC, Castracane VD. Leptin in pregnancy: an update. Biol Reprod 2006;74:218-229.

12. Matjila M, Millar R, van der Spuy $Z$, et al. The differential expression of Kiss1, MMP9 and angiogenic regulators across the feto-maternal interface of healthy human pregnancies: implications for trophoblast invasion and vessel development. PLoS ONE 2013;8:e63574.

13. Loegl J, Nussbaumer E, Hiden U, et al. Pigment epithelium-derived factor (PEDF): a novel trophoblast-derived factor limiting feto-placental angiogenesis in late pregnancy. Angiogenesis 2016;19:373-388.

14. Skupien J, Cyganek K, Malecki MT. Diabetic pregnancy: an overview of current guidelines and clinical practice. Curr Opin Obstet Gynecol 2014;26:431-437.

15. Jirkovska M, Kubinova L, Janacek J, et al. Topological properties and spatial organization of villous capillaries in normal and diabetic placentas. J Vasc Res 2002;39:268-278.

16. Mayhew TM, Sorensen FB, Klebe JG, et al. Growth and maturation of villi in placentae from well-controlled diabetic women. Placenta 1994;15:57-65.

17. Younes B, Baez-Giangreco A, al-Nuaim L, et al. Basement membrane thickening in the placentae from diabetic women. Pathol Int 1996;46: 100-104.

18. Calderon IM, Damasceno DC, Amorin RL, et al. Morphometric study of placental villi and vessels in women with mild hyperglycemia or gestational or overt diabetes. Diabetes Res Clin Pract 2007;78:65-71.

19. Taricco E, Radaelli T, Rossi G, et al. Effects of gestational diabetes on fetal oxygen and glucose levels in vivo. BJOG 2009;116:1729-1735.

20. Cvitic S, Desoye G, Hiden U. Glucose, insulin, and oxygen interplay in placental hypervascularisation in diabetes mellitus. Biomed Res Int 2014;2014:145846.

21. International Association of D, Pregnancy Study Groups Consensus $P$, Metzger BE, et al. International association of diabetes and pregnancy study groups recommendations on the diagnosis and classification of hyperglycemia in pregnancy. Diabetes Care 2010;33: 676-682.

22. Chen $B$, Longtine MS, Sadovsky $Y$, et al. Hypoxia downregulates p53 but induces apoptosis and enhances expression of BAD in cultures of human syncytiotrophoblasts. Am J Physiol Cell Physiol 2010;299: C968-C976. 
23. Cervar M, Blaschitz A, Dohr G, et al. Paracrine regulation of distinct trophoblast functions in vitro by placental macrophages. Cell Tissue Res 1999;295:297-305.

24. Lang I, Schweizer A, Hiden U, et al. Human fetal placental endothelia cells have a mature arterial and a juvenile venous phenotype with adipogenic and osteogenic differentiation potential. Differentiation 2008;76:1031-1043.

25. Bach LA. Endothelial cells and the IGF system. J Mol Endocrinol 2015;54:R1-13.

26. Dunn IF, Heese O, Black PM. Growth factors in glioma angiogenesis: FGFs, PDGF, EGF, and TGFs. J Neurooncol 2000;50:121-137.

27. Carmeliet $P$, Jain RK. Molecular mechanisms and clinical applications of angiogenesis. Nature 2011;473:298-307.

28. Yang X, Meyer K, Friedl A. STAT5 and prolactin participate in a positive autocrine feedback loop that promotes angiogenesis. J Biol Chem 2013;288:21184-21196.

29. Adams JC, Lawler J. The thrombospondins. Cold Spring Harb Perspect Biol 2011;3:a009712.

30. Golzar F, Javanmard SH. The effects of kisspeptin-10 on migration and proliferation of endothelial cell. Adv Biomed Res 2015:4:41.

31. Das AM, Seynhaeve AL, Rens JA, et al. Differential TIMP3 expression affects tumor progression and angiogenesis in melanomas through regulation of directionally persistent endothelial cell migration. Angiogenesis 2014;17:163-177.

32. Parissis J, Filippatos G, Adamopoulos S, et al. Hematopoietic colony stimulating factors in cardiovascular and pulmonary remodeling: promoters or inhibitors? Curr Pharm Des 2006;12:2689-2699.
33. Tahergorabi Z, Khazaei M. Leptin and its cardiovascular effects: Focus on angiogenesis. Adv Biomed Res 2015;4:79.

34. Rosenkilde MM, Schwartz TW. The chemokine system - a major regulator of angiogenesis in health and disease. APMIS 2004;112: 481-495.

35. Bussolino F, Di Renzo MF, Ziche M, et al. Hepatocyte growth factor is a potent angiogenic factor which stimulates endothelial cell motility and growth. J Cell Biol 1992;119:629-641.

36. Fajardo LF, Kwan HH, Kowalski J, et al. Dual role of tumor necrosis factor-alpha in angiogenesis. Am J Pathol 1992;140:539-544.

37. Holash J, Maisonpierre PC, Compton D, et al. Vessel cooption, regression, and growth in tumors mediated by angiopoietins and VEGF. Science 1999;284:1994-1998.

38. Lobov IB, Brooks PC, Lang RA. Angiopoietin-2 displays VEGFdependent modulation of capillary structure and endothelial cell survival in vivo. Proc Natl Acad Sci USA 2002;99:11205-11210.

39. Zhang SX, Wang JJ, Gao G, et al. Pigment epithelium-derived factor downregulates vascular endothelial growth factor (VEGF) expression and inhibits VEGF-VEGF receptor 2 binding in diabetic retinopathy. J Mol Endocrinol 2006;37:1-12.

40. Autiero $\mathrm{M}$, Waltenberger J, Communi $\mathrm{D}$, et al. Role of PIGF in the intraand intermolecular cross talk between the VEGF receptors Flt1 and Flk1. Nat Med 2003;9:936-943.

41. Dimasuay KG, Boeuf $P$, Powell TL, et al. Placental responses to changes in the maternal environment determine fetal growth. Front Physiol 2016;7:12. 